\title{
Precompetition injury and subsequent tournament performance in full-contact taekwondo
}

\author{
Michael Feehan and Anna E Waller* \\ Faculty of Social and Behavioral Sciences, Department of Health Policy and Management, Johns Hopkins \\ University School of Hygiene and Public Health, Baltimore, Maryland, USA; ${ }^{*}$ Department of Emergency \\ Medicine, School of Medicine, University of North Carolina at Chapel Hill, North Carolina, USA
}

\begin{abstract}
Objective - To determine the pattern of injuries sustained by taekwondo competitors, and how previous injury affects performance in sporting competition.

Methods - Competitors at a major New Zealand fullcontact taekwondo tournament were given a comprehensive questionnaire immediately before competition. Self reports of injuries sustained through taekwondo in the previous 12 months were determined.

Results - No significant sex differences were found. The injury prevalence was high, but comparatively less than reported for other contact sports such as rugby football. However, one in 20 injuries was a closed head injury. No significant associations between prior injury and tournament outcome were found.

Conclusions - Taekwondo can be a source of disabling injury. However, the fact that prior injury did not appear to be associated with impaired competition performance may militate against acceptance of control and regulation by competitors and coaches.

(Br J Sports Med 1995; 29: 258-262)
\end{abstract}

Keywords : taekwondo; injury; performance; regulation

Taekwondo is the national martial art and sport of Korea with origins dating back at least 1000 years. ${ }^{1-3}$ Given its strong emphasis on sporting performance, taekwondo in its modern form is an increasingly popular sport worldwide. It is practised in at least 140 countries, and 120 nations are official members of the sports major organising body, the World Taekwondo Federation (WTF). The WTF was admitted to the International Olympic Committee in 1980, and taekwondo was a demonstration sport in the 1988 and 1992 Olympic games. ${ }^{3} 4$

In addition to traditional training in patterns (forms) and prearranged non-contact or semi-contact sparring routines, taekwondo students participate in full-contact tournaments. At these tournaments competitors wear protective equipment: head gear, a chest and abdomen protector, a groin guard, and shin and forearm guards. Competitors fight in divisions according to age, gender, experience, and weight. Fights in the black belt divisions

Address for correspondence: Dr Michael Feehan, Faculty of Social and Behavioral Sciences, Department of Health Policy and Management, Johns Hopkins University School of Hygiene and Public Health, 7th Floor Hampton House, 624 N Broadway, Baltimore, Maryland 21205. USA generally last three rounds, each round lasting three minutes. A fight can be won by knockout or on points. Punching is not permitted to the head but kicks to the head and face are quite legal and are clear point scoring techniques.

Although the perception among practitioners is that major injuries are uncommon, few data on injuries sustained through taekwondo are available. ${ }^{5}$ This is despite a heavy emphasis on kicking techniques. In kinetic studies, taekwondo kicks have been shown to have very high injury potential. ${ }^{7}$ What is known about taekwondo injuries, and martial arts injuries in general, has come from studies focused on injuries sustained during tournament competition or precompetition trials. ${ }^{5-9}$ However, it is estimated that $60 \%$ of injuries sustained in competition and practice are not reported. ${ }^{10}$

During the 6th Taekwondo World Championships, $4 \%$ of competitors were admitted to casualty wards with injuries, the most severe being head and neck trauma. ${ }^{8}$ In a study by Oler $e t$ al. of two National US Taekwondo tournaments (one senior and one junior) injuries to the head and neck were the most common reported to on-site medical personnel. ${ }^{5}$ Calculated from the data presented in Oler's paper, it appears that 23 of approximately 700 adult competitors sustained head or neck injuries $(33 / 1000)$ and 49 of around 3000 junior competitors (16/1000).

Among 48 male and 48 female competitors at the 1988 US Olympic Team Trials, the injury rate for men (12.74/100 athlete-exposures) was $40 \%$ higher than that for women (9.01/100). ${ }^{7}$ Most injuries were to the head or foot. As in Oler's study, ${ }^{5}$ these rates were calculated for injuries reported to on-site medical staff and will be an underestimate of the total injuries sustained during the tournament. Even though treatment was sought, most injuries were not of sufficient severity to keep the athlete from further competition. Applying a more stringent criterion of injury severity resulted in $15 \%$ of the injuries experienced by both men and women being classed as 'time loss' injuries (an injury that requires one or more days away from practice or competition).

The aims of the present study were: (1) to determine the pattern of injuries sustained by taekwondo competitors in the 12 months before a competition; and (2) to determine how prior injury experience may affect 
individual performance in a sporting competition where indicators of success would be easily quantifiable and unambiguous (fight wins and losses). It was hypothesised that those with fewer precompetition injuries would have improved competition success.

\section{Methods}

\section{Sample and design}

Sample members were competitors at the two day 1993 South Island Taekwondo Championships of the New Zealand Taekwondo Federation, Inc (NZTF). At the time of the competition about 5000 players belonged to the 150 member clubs of the NZTF, which is affiliated with the WTF and the New Zealand Olympic and Commonwealth Games Association. The South Island and North Island Championships are the two major regional competitions held before the New Zealand National Championships.

MF introduced the study during the opening ceremony on the morning of the first day. The sample was restricted to competitors 16 years old and over, but otherwise was open to males and females of all competing ranks. Prospective competitors were handed a package containing a consent form, a questionnaire, a pen, and an envelope. The competitors were invited to complete the questionnaire as soon as possible (that is, before their first fight), enclose it in the envelope and place the sealed envelope in a box at the main entrance of the hall. Staff of the University of Otago Injury Prevention Research Unit were on hand to answer questions and provide assistance as required.

The analyses presented here are restricted to the 48 competitors who had at least one fight during the tournament and who completed the questionnaire: 39 males $(81 \%)$ and nine females $(18 \%)$, who ranged in age from 16 to 45 years (mean $=22.2$, SD 5.71). The majority of the sample identified themselves as European $(83 \%)$, and the remainder indicated their ethnicity as Chinese ( $8 \%$ ), Maori $(4 \%)$, and Pacific Islander $(4 \%)$. A large proportion were students at either the tertiary $(25 \%)$ or secondary level $(14 \%)$. A large proportion had experienced a period of unemployment in the last year (35\%), with an average of 4.5 months (SD 4.08), and $41 \%$ reported that it was 'somewhat' or 'very difficult' to support themselves financially at the moment. A fifth of the sample was married (18\%), and none of the female competitors reported being pregnant at the time of competition.

\section{Measures}

The questionnaire administered was a modified version of the preseason questionnaire used in the New Zealand rugby injury and performance project. This is a prospective longitudinal study of a cohort of male and female rugby players being conducted by the University of Otago Injury Prevention Research Unit and the Human Performance Centre of the School of Physical Education. ${ }^{11}$ The questionnaire comprised six sections: general background; taekwondo and other sport experience; specific tournament preparation; taekwondo related and other injury experience; health, lifestyle, and wellbeing; and attitudes and opinions about taekwondo and sports in general.

Taekwondo injury experience was assessed by providing a list of commonly occurring sports injuries with the instruction that an injury was 'defined as a physical problem that caused you to miss (a) at least one taekwondo practice of scheduled training session OR (b) required at least one visit to a health professional for treatment.' Competitors checked all injuries that they had experienced in the previous 12 months as a result of taekwondo participation. This was followed by a list of injuries experienced in the previous year that required assistance from a health professional, in addition to those reported for taekwondo participation. Competitors were also asked if they had current injuries affecting their training ability or chronic long term injuries requiring special safety gear (for example, strapping or brace). They were also asked if they had used anything to help them train or compete while recovering from injury, if they had performed taekwondo against medical advice, and if they had difficulties obtaining medical advice for sporting injuries.

\section{Results}

The analyses presented here are largely categorical tests of association. Given the comparatively small numbers and the likelihood of obtaining cell frequencies of less than five, Fisher's exact test results are reported rather than the more approximate $\chi^{2}$ statistic. $^{12}{ }^{13}$

\section{Taekwondo and competition experience}

In terms of taekwondo background, the average age that competitors took up the sport was 16-17 years, although a large proportion took it up at age 20 or older $(32 \%)$. Regarding rank, $40 \%$ of the sample were competing in the 'beginner colour-belt' division, $25 \%$ in the 'intermediate colour-belt' division, and $34 \%$ in the 'black belt' division. Of the black belt competitors, the majority were 1st Dan (69\%) and the remainder were 2nd Dan. Given the relatively small sample, analyses for rank were conducted with the dichotomy of black belt and above versus all lower ranks. Females were significantly over-represented at the senior level: $67 \%$ of the females fought in the black belt division compared with $26 \%$ of the males (Fisher's exact test, $P=0.045)$. Over half of the competitors had fought previously in an NZTF full-contact tournament (53\%). There was no significant difference in previous NZTF competition between males and females (Fisher's exact test, $P=0.470$ ). As would be expected, rank was significantly associated with prior NZTF tournament participation (Fisher's exact test, $P=0.0001$ ); $94 \%$ of the black belt competitors had fought previously compared with $32 \%$ of the lower ranks. Overall, $79 \%$ considered taekwondo to be their major sport, with no significant sex difference (Fisher's exact test, $P=1.000$ ). Taekwondo was the major sport for all at the black belt level compared with $73 \%$ of those at the lower levels, a significant difference (Fisher's exact test, $P=0.037$ ). 
Table 1. Body sites of taekwondo related injuries experienced in the previous 12 months

\begin{tabular}{lcr}
\hline Site & $n$ & $\%$ \\
\hline Head & 8 & 10 \\
Neck & 1 & 1 \\
Shoulder/collarbone & 8 & 10 \\
Upper or lower back & 9 & 11 \\
Chest or abdomen & 5 & 6 \\
Wrist, hand, or digits & 6 & 7 \\
Upper or lower leg & 27 & 35 \\
Ankle, foot, or digits & 13 & 16 \\
Total & $77^{*}$ & \\
\hline
\end{tabular}

* Two injury sites were unspecified

Table 2. Types of taekwondo related injuries experienced in the previous 12 months

\begin{tabular}{lrr}
\hline Type of injury & $n$ & $\%$ \\
\hline Sprain, strain, other soft tissue & 65 & 82 \\
Fracture & 6 & 7 \\
Closed head injury & 4 & 5 \\
Laceration or graze & 3 & 3 \\
Dislocation & 1 & 1 \\
Total & 79 & \\
\hline
\end{tabular}

\section{Precompetition injury experience}

In the 12 months before the competition, over two thirds $(69 \%)$ of the competitors had experienced at least one taekwondo related injury of sufficient severity to make them miss training or seek treatment from a health professional. The injury prevalence estimates were virtually identical for males $(69 \%)$ and females $(67 \%)$; Fisher's exact test, $P=1.000$. The differences in injury prevalence according to rank (black belt or lower) were also not significant; Fisher's exact test, $P=0.202$. Of those who had been injured, $27 \%$ had sustained one injury, $42 \%$ had sustained two, and $30 \%$ a total of three or more. The maximum number of injuries sustained was six, experienced by one individual. In total, 79 separate injuries were reported, giving an average of 2.3 injuries per injured competitor.

As shown in Table 1 , the most common site of injury was the upper or lower leg, followed by the ankle or foot. Nine injuries (11\%) were to the head or neck. Table 2 describes the injuries by type. The vast majority comprised soft tissue damage such as haematoma and sprains. Fractures, closed head injury (concussion), cuts, and dislocations were much less common.

Over the previous 12 months, $37 \%$ of the sample had experienced at least one injury from an activity other than taekwondo which required medical assistance. No significant differences were found in the proportion of these injuries by sex (Fisher's exact test, $P=1.000$ ) or rank (Fisher's exact test, $P=0.206$ ). A total of 31 nontaekwondo-related injuries were reported, giving an average of 1.7 per injured person. As with the taekwondo related injuries, sprains and other soft tissue injuries predominated $(75 \%)$, followed by fractures (17\%). The most common sites for non-taekwondo- related injuries were the lower limbs ( $52 \%$ ), followed by the upper limbs (19\%), and head, neck, and collarbone (16\%).

It was thought that those injured through taekwondo may also be more likely to experience injury generally (for example, through other sports participation or risk taking activities). Although $46 \%$ of those with a taekwondo related injury had sustained an injury from another activity compared with $20 \%$ of those without a taekwondo injury, the difference in proportions failed to reach significance (Fisher's exact test, $P=0.177$ ).

In terms of the competitor's injury status on the day of competition, 17 individuals (35\%) reported that they had a current injury that was affecting their ability to perform, and three of those competitors required special strapping or other support to enable performance. Seven individuals $(15 \%)$ reported that they had used something to help them continue taekwondo training while recovering from an injury (principally the use of ultrasound therapy and the taking of analgesic medication). Eight competitors ( $17 \%$ ) reported that they had continued to train or compete against specific medical advice. Nine individuals (19\%) reported they had difficulty obtaining medical advice for sporting injuries.

\section{Tournament outcome}

In some weight/experience divisions with a small number of competitors, the opening round was also the final. In other divisions, competitors had up to two fights before their final. For this paper, each individual's success or failure at the competition was defined in terms of their official first round win or loss record. This gave 24 competitors with a first round win, and the same number with a first round loss and elimination. Fight outcome was not significantly associated with age in years $(t=0.30,46 d f, P=0.7652)$. A series of bivariate analyses were conducted to determine if fight outcome were associated with prior injury experience and other characteristics. As shown in Table 3, fight outcome was not significantly associated with any of the following: a history of any taekwondo related injury in the previous 12 months; any other nontaekwondo-related injury experienced in the previous 12 months; and a current injury at competition entry. It was also considered that a personal style whereby individuals did not allow injury to stop them competing may have been related to outcome. The competitors who used something to help them compete or train while injured and those who had a history of performing taekwondo against medical advice were no more or less likely to win their opening round. Fight success was also not associated with a history of previous NZTF tournament experience.

\section{Discussion}

Birrer $e$ al.$^{14}$ asserted that 'Tae Kwon Do is a safe sport - only 1 in 500 injuries is serious.' However, Oler et al. ${ }^{5}$ have been critical of this view. In response to Birrer and Halbrook, ${ }^{15}$ who asserted that 'all forms of martial arts are safe', Oler et al. argued that the martial arts' 
Table 3. First round fight outcome in relation to possible factors associated with wins or losses

\begin{tabular}{|c|c|c|c|}
\hline & \multicolumn{2}{|c|}{ Fight outcome } & \multirow[b]{2}{*}{$\begin{array}{c}\text { Fisher's Exact test } \\
(P)\end{array}$} \\
\hline & $\begin{array}{c}\text { Win } \\
(n=24)\end{array}$ & $\begin{array}{c}\text { Loss } \\
(n=24)\end{array}$ & \\
\hline History of taekwondo injury in previous 12 months & $17(71 \%)$ & $16(67 \%)$ & 1.000 \\
\hline Other non-taekwondo injury in previous 12 months & $8(33 \%)$ & $10(42 \%)$ & 0.766 \\
\hline Current injury at competition entry & $11(46 \%)$ & $6(25 \%)$ & 0.227 \\
\hline History of using something to compete or train while injured & $3(13 \%)$ & $4(17 \%)$ & 1.000 \\
\hline History of performing taekwondo against medical advice & $3(13 \%)$ & $5(21 \%)$ & 0.701 \\
\hline Prior NZFT tournament experience & $12(50 \%)$ & $13(57 \%)$ & 0.772 \\
\hline
\end{tabular}

Note: A total of 47 competitors provided information about performing against medical advice and 46 provided information about prior NZTF tournament experience.

transition from a means to kill and disable to a sport was 'incomplete', and some of the injury risks in the martial arts were unwarranted and preventable. The results of the present study would tend to contradict the assertion of writers such as Birrer et al. ${ }^{14}$ that taekwondo is a safe sport. No sport should be considered 'safe' if seven out of 10 competitors (as in the present study) had received an injury in the previous 12 months which was severe enough to preclude training or require medical assistance. Although the present study confirmed the findings of other studies that contusions, sprains, and strains to the lower extremities were the most common taekwondo related injuries, ${ }^{74}$ one in 20 injuries was a closed head injury - further evidence that taekwondo is a source of potentially disabling injury.

However, the results from the present study do need to be interpreted in relation to other sporting activities. Zemper and Pieter ${ }^{7}$ found that among eight collegiate sports, the 'time loss' injury rate for males (23.6/1000 athlete-exposures) placed taekwondo third after wrestling (31.8) and American football (31.0) as a source of competition injury, and ahead of soccer (18.7) and ice hockey (15.7). Among seven sports, the women's injury rate for taekwondo (13.5) was ranked third after gymnastics (18.1) and soccer (15.3), and ahead of field hockey (7.2) and lacrosse (7.0).

Because the measures used in this study were modified from those used in the New Zealand rugby injury and performance project (RIPP), ${ }^{11}$ contrasting the present data with the one year prevalence data obtained on rugby related injuries is an ideal comparison. Gerrard et al. reported that $84.4 \%$ of male rugby players and $59.6 \%$ of the female players in the RIPP study experienced at least one rugby related injury in the previous year. ${ }^{16}$ Thus, at least for males, full-contact taekwondo (where practitioners train explicitly to knock out opponents with high kicking combinations), while not 'safe', is at least safer than rugby (a contact sport in which striking the opponent with the intent to injure is illegal).

Interestingly, the difference in injury prevalence between males and females in the RIPP study was significant, but no significant sex differences were found in the present taekwondo study. There may be many factors which could account for this (for example, relative to males, female rugby players have different exposure rates, or differences in technique when playing the game), but one intriguing possibility is that a significant proportion of rugby injuries may in fact be intentional, foul behaviour perhaps being more common among male players. Taekwondo may be safer than a sport such as rugby because the competition environment is more controlled, with the referee contending with only two players rather than 30 . The extent of intentional injury in the game of rugby is one avenue of study currently being conducted in the RIPP.

Another possibility is that the lower level of injuries compared to rugby may be a result of the extensive protective equipment worn in taekwondo competition. However, it should be kept in mind that the present data reflect a year of training. Taekwondo participants often train (and sustain injuries) at the 'no contact' or 'light contact' levels which have less stringent requirements for the use of protective equipment. There is also some evidence to suggest that some martial arts equipment serves to protect the attacker rather than the defender, allowing for greater force than would otherwise be applied. $^{5}$,

The findings of this study with regard to the prediction of tournament success is of critical importance to those interested in developing injury control strategies and preventive interventions. Largely on the basis of descriptive studies, several investigators have made blanket recommendations for the prevention of injury in taekwondo and the martial arts in general. These have included: banning spinning kicks to the head, ${ }^{17}$ banning head contact for children and limiting it to certain experienced adults, ${ }^{5}$ and banning kicks to the face. ${ }^{8}$ As Oler et al. pointed out, 'condemnation and attempts to ban this activity will probably be less productive than attempts at regulation'. ${ }^{5}$ If preventive efforts through regulation are to be implemented, changes must be seen as desirable by both competitors and administrators.

From the perspective of competitors and coaches, it is more likely that regulation would be accepted if it could be clearly shown that prior injury had a deleterious effect on performance. However, this was not the case in the present study: none of the injury related variables was significantly associated with fight success or failure. These findings were unexpected and warrant replication. It may be that the use of only first round 
outcome measures does not give sufficient discriminatory power. In a larger study, for example at a national level competition, there may be sufficient statistical power to analyse final-round-only outcome measures (where all competitors must have had preliminary bouts).

It is also possible that those who had received the most severe injuries during the lead-up to the competition did not enter, or may even have dropped out of the sport altogether. Given the growing popularity of martial arts training, further research in this area is needed, and the application of prospective methodology, such as that successfully used in the RIPP study, may be ideal. ${ }^{11} \mathrm{~A}$ cohort of taekwondo students could thus be followed throughout the year in order to obtain detailed information obtained about injury experience, competition and training exposure, periods of nonparticipation, and drop-out.

\section{Acknowledgements}

We would like to thank Grandmaster Jung Nam Lee (10th Dan), President of the New Zealand Taekwondo Federation Inc, for his personal interest and the support of the NZTF for this study. Thanks are expressed to Mr Matt Ransom (4th Dan), organiser of the 1993 South Island Taekwondo Championships, for his assistance in gathering data and his advice throughout the project. For their assistance during the tournament and their comments on earlier versions of the manuscript, we would like to thank Mr Steve Marshall, Mr Ken Quarrie, and Dr Dave Chalmers (Deputy Director, Injury Prevention Research Unit). The study was conducted while the authors were employed in the rugby injury and performance project, funded by a grant from the Accident Rehabilitation and Compensation Insurance Corporation (ACC). Analyses were conducted while MF was the recipient of a Health Research Council of New Zealand overseas research fellowship. The University of Otago's Injury Prevention Research Unit is funded by the Health Research Council of New Zealand and the ACC. The views expressed in this paper are those of the authors and do not necessarily reflect those of the above organisations.

\section{References}

1 Cho SH. Korean karate: free fighting techniques. Rutland VT: Charles E Tuttle, 1968.

2 Chun R. Advancing in tae kwon do. New York: Harper and Row, 1982.

3 Park YH, Park YH and Gerrard J. Tae kwon do: the ultimate reference guide to the world's most popular martial art. New York: Facts on File Publications, 1989.

4 Lucas J. Taekwondo, pelote Basque/jai-alai, and roller hockey three unusual Olympic demonstration sports. $J$ Phys Educ Recreation Dance 1992, April: 80 - 82.

5 Oler M, Tomson W, Pepe H, Yoon D, Branoff R, Branch, J. Morbidity and mortality in the martial arts: a warning. J Trauma 1991; 31: $251-3$.

6 Serina ER, Lieu ER. Thoracic injury potential of basic competition taekwondo kicks. J Biomech 1991; 10: $951-60$.

7 Zemper ED, Pieter W. Injury rates during the 1988 US Olympic Team Trials for taekwondo. Br J Sports Med 1989; 23 : $161-4$.

8 Siana JE, Borum R, Kryger H. Injuries in taekwondo. Br J Sports Med 1986; 20 : $165-6$.

9 Johannsen HV, Noerregaard FOH. Prevention of injuries in karate. Br J Sports Med 1988; 22 : 113 - 5.

10 Birrer RB, Birrer CD. Unreported injuries in the martial arts. $\mathrm{Br} J$ Sports Med 1983; 17: $131-4$.

11 Waller AE, Feehan M, Marshall SW, Chalmers DJ. The New Zealand Rugby Injury and Performance Project: I. Design and methodology of a prospective follow-up study. Br J Sports Med $1994 ; 28: 223-8$.

12 Fisher RA. Statistical methods for research workers. Edinburgh: Oliver and Boyd, 1941.

13 Snedecor GW, Cochran WG. Statistical methods, 7th ed. Ames IA: Florida State University Press, 1980.

14 Birrer RB, Birrer C, Son DS, Stone D. Injuries in tae kwon do. Physician Sports Med 1981; 9: 97 - 103.

15 Birrer RB, Halbrook SP. Martial arts injuries. Am J Sports Med $1988 ; 16: 408-10$.

16 Gerrard DF, Waller AE, Bird YN. The New Zealand Rugby Injury and Performance Project: II. Previous Injury Experience of a Rugby Playing Cohort. Br J Sports Med 1994; 28: 229-33.

17 McLatchie G. Karate and karate injuries. Br J Sports Med 1981; 15 : $84-6$. 